\title{
COMPUTATION OF A TEST STATISTIC IN DATA QUALITY CONTROL
}

\author{
XIAO-WEN CHANG*, CHRISTOPHER C. PAIGE ${ }^{\dagger}$, AND CHRISTIAN C.J.M. TIBERIUS $\ddagger$
}

\begin{abstract}
When processing observational data, statistical testing is an essential instrument to hopefully render harmless incidental anomalies and disturbances in the measurements. A commonly used test statistic based on the general linear model is the generalized likelihood ratio test statistic. The standard formula given in the literature for this test statistic is not defined if the noise covariance matrix is singular, and is not suitable for computation if any of the matrices involved is ill-conditioned. Based on Paige's generalized linear least squares method [Comm. Statist., Vol. B7, 437-453, 1978], a numerically stable approach is proposed for the computation of the test statistic, as well as for the estimates of the parameter vectors, and reliable representations of the error covariance matrices for these estimates are presented. This approach allows the noise covariance matrix to be singular, and can be applied directly to the linear model with linear equality constraints.
\end{abstract}

Key words. Data quality control, statistical testing, generalized likelihood ratio, generalized least squares, generalized QR factorization, numerical stability.

AMS subject classifications. 62J12, 62J20, 65F $20,65 \mathrm{~F} 25$

1. Introduction. For a very wide range of applications, data processing involves least squares (LS) estimation. The LS estimate in itself, however, does not offer any indication of the validity of the result. Once some basic conditions are met, LS estimates for the unknown parameters, for example the position coordinates in a Global Positioning System (GPS) application, are computed. Usually these give no indication as to whether, or to what extent, the input measurements are corrupted, i.e., do not satisfy the assumed mathematical model. In critical applications such as real-time navigation, the user has to be protected against misleading (position) information, caused by one or more anomalies in the measurements underlying the resulting position estimate. The incoming data need to be carefully validated by means of statistical testing, using the redundancy in the measurement set-up and the consequent strength of the mathematical model. Only then can the integrity of the eventual position solution be assured. A discussion of statistical testing for data quality control can be found for example in [11, Chap. 7] (mainly for GPS data processing) and [12].

A commonly used general test statistic for model validation based on linear models is called the generalized likelihood ratio test statistic, which follows the Chi-square distribution. A direct evaluation of the standard formula for the test statistic (see (2.8)) may lead to large rounding errors in the result when any of the matrices involved is ill-conditioned (i.e., has a large ratio of maximum to minimum singular value). In particular, those methods which use the inverse of the covariance matrix are numerically unstable, and so can lead to large errors with finite precision computation. This was illustrated by example in $[5, \S 4]$. Furthermore, the formula is not

*School of Computer Science, McGill University, Montreal, Quebec, Canada, H3A 2A7, (chang@cs.mcgill.ca). The research of this author was supported by NSERC of Canada grant RGPIN217191-03 and FCAR of Quebec, Canada grant 2001-NC-66487.

†School of Computer Science, McGill University, Montreal, Quebec, Canada, H3A 2A7, (paige@cs.mcgill.ca). Research supported by NSERC of Canada Grant RGPIN9236-01.

${ }^{\ddagger}$ Section of Mathematical Geodesy and Positioning, Delft University of Technology, Thijsseweg 11, 2629 JA Delft, The Netherlands, (c.c.j.m.tiberius@geo.tudelft.nl). 
defined when the noise covariance matrix is singular. To our knowledge, there has not yet been a numerically sound approach for computing this test statistic. The goal of this paper is to present a numerically stable algorithm to compute the test statistic and the estimates of the parameter vectors as well, based on Paige's generalized linear least squares method (see [5]-[8]). Our approach can handle the case where the noise covariance matrix is singular. This will also allow us to deal with linear models with linear equality constraints without altering the algorithm.

It appears unlikely that a very ill-conditioned noise covariance matrix will arise directly in standard GPS applications. But constraints do appear, and these have been handled by introducing an additional noise vector whose covariance matrix has very small diagonal elements, leading to an inexact model whose noise covariance matrix is very ill-conditioned. Ill-conditioned or singular noise covariance matrices can of course appear in other applications.

This paper is organized as follows. In Section 2 we introduce the general linear model and derive the test statistic, then Section 3 shows the dangers of some obvious ways of computing this test statistic. In Section 4 we first reformulate the optimization problems for estimating the parameter vectors and give an equivalent formula for the test statistic, then present an algorithm for computing the test statistic and the estimates of the parameter vectors as well, and give reliable representations of the error covariance matrices for the estimates. The backward numerical stability of the algorithm is proven in Section 5. In Section 6 we show how to compute the test statistic and the estimates of parameter vectors when the noise covariance matrix may be singular. We also illustrate how linear equality constraints result in a model which effectively has a singular noise covariance matrix, so that the method here can be applied directly. Finally we give a brief summary in Section 7 .

Throughout this paper we work with reals only. $\Re^{m}$ denotes the class of real $m$-dimensional vectors, while $\Re^{m \times n}$ denotes the class of real $m \times n$ matrices. $\mathcal{R}(A)$ denotes the range of the matrix $A$. The identity matrix is denoted by $I$ and its $i$-th column by $e_{i}$. For a matrix $A, A^{\dagger}$ denotes its Moore-Penrose generalized inverse. We write $\|x\|_{2}=\sqrt{x^{T} x}$ for vectors and $\|A\|_{F}=\sqrt{\sum_{i j} a_{i j}^{2}}$ for matrices. We use $\mathcal{E}\{\cdot\}$ to denote the expected value, and $\operatorname{cov}\{\cdot\}$ to denote the covariance, that is $\operatorname{cov}\{x\}=$ $\mathcal{E}\left\{(x-\mathcal{E}\{x\})(x-\mathcal{E}\{x\})^{T}\right\}$ for a random vector $x$. If $v$ is a normally distributed random vector with mean $\bar{v}$ and covariance $\sigma^{2} V$, we write $v \sim \mathcal{N}\left(\bar{v}, \sigma^{2} V\right)$. $V$ is sometimes called the cofactor matrix in geodesy, see for example [3, p. 250]. If a random variable $\delta$ follows a Chi-square distribution with non-centrality parameter $\lambda$ and $q$ degrees of freedom, we write $\delta \sim \chi^{2}(q, \lambda)$.

2. Linear model and test statistic. Suppose we have a linear model

$$
y=A x+v, \quad v \sim \mathcal{N}\left(0, \sigma^{2} V\right),
$$

where $y \in \Re^{m}$ is a random measurement vector, $A \in \Re^{m \times n}$ with $m \geq n$ is a design matrix with full column rank, $x \in \Re^{n}$ is an unknown parameter vector, and $v \in \Re^{m}$ is a random noise vector following a normal distribution with zero mean and a known symmetric nonnegative definite covariance matrix $\sigma^{2} V$.

The above model may, however, not reflect reality properly in all cases. The model is misspecified when for instance one or more measurements are corrupted by outliers. They will invalidate the results of estimation. To safeguard against anomalous measurements, it is important to validate the data and model used. Here we restrict the misspecification to the mean of the measurement vector $y$, i.e. an 
error of additive nature. Suppose (2.1) above represents the working model, the null hypothesis $\mathrm{H}_{0}$. In a general form the alternative hypothesis $\mathrm{H}_{a}$ then reads

$$
y=A x+C \nabla+v, \quad v \sim \mathcal{N}\left(0, \sigma^{2} V\right),
$$

where the known matrix $C \in \Re^{m \times q}$ with $1 \leq q \leq m-n$ specifies the type of model error that occurred, $[A, C]$ has full column rank, and $\nabla \in \Re^{q}$ is an unknown constant vector (see for example [12, Sec 4.1]). One special case which leads to the so-called w-test statistic is where the cofactor matrix $V$ is the identity matrix, and there is only a possible outlier in the $i$-th measurement, i.e. $C=e_{i}$. If we take $i=1, \ldots, m$, we will have $m$ alternative hypotheses. For other special cases which may arise from GPS, see [11, p. 280].

Initially we assume that $V$ is nonsingular. Following [12, Sec 4.1], we briefly derive the generalized likelihood ratio test statistic for testing the null hypothesis $\mathrm{H}_{0}$ against the alternative hypothesis $\mathrm{H}_{a}$.

Let $p(y \mid x)$ and $p(y \mid x, \nabla)$ denote the density functions of $y$ under $\mathrm{H}_{0}$ and $\mathrm{H}_{a}$, respectively. Then

$$
\begin{aligned}
p(y \mid x) & =\frac{1}{(2 \pi)^{\frac{m}{2}} \sigma \operatorname{det}(V)^{\frac{1}{2}}} \exp \left[-\frac{1}{2 \sigma^{2}}(y-A x)^{T} V^{-1}(y-A x)\right] \\
p(y \mid x, \nabla) & =\frac{1}{(2 \pi)^{\frac{m}{2}} \sigma \operatorname{det}(V)^{\frac{1}{2}}} \exp \left[-\frac{1}{2 \sigma^{2}}(y-A x-C \nabla)^{T} V^{-1}(y-A x-C \nabla)\right] .
\end{aligned}
$$

Let $x_{0}$ be the maximum likelihood estimator of $x$ under $\mathrm{H}_{0}$, and let $\left\{x_{a}, \nabla_{a}\right\}$ be the maximum likelihood estimator of $\{x, \nabla\}$ under $\mathrm{H}_{a}$, respectively, i.e.,

$$
p\left(y \mid x_{0}\right)=\max _{x} p(y \mid x) \leq p\left(y \mid x_{a}, \nabla_{a}\right)=\max _{x, \nabla} p(y \mid x, \nabla) .
$$

We expect these values to be comparable under $\mathrm{H}_{0}$. Obviously $x_{0}$ and $\left\{x_{a}, \nabla_{a}\right\}$ are respectively the solutions of the following two generalized linear least squares (GLLS) problems (also referred to as weighted linear least squares problems in some literature):

$$
\begin{array}{ll}
\operatorname{GLLS}_{0}: & \min _{x}(y-A x)^{T} V^{-1}(y-A x) ; \\
\operatorname{GLLS}_{a}: & \min _{x, \nabla}(y-A x-C \nabla)^{T} V^{-1}(y-A x-C \nabla) .
\end{array}
$$

The two estimators are also the best linear unbiased estimators (BLUE) of $x$ under $\mathrm{H}_{0}$ and $\{x, \nabla\}$ under $\mathrm{H}_{a}$, respectively (see for example [4, Sec 3.2]). For later use, we define the corresponding residuals

$$
r_{0} \equiv y-A x_{0}, \quad r_{a} \equiv y-A x_{a}-C \nabla_{a} .
$$

The generalized likelihood ratio is defined to be (see for example [12, Sec 4.1])

$$
\mathcal{L}\left(\mathrm{H}_{0}, \mathrm{H}_{a}\right) \equiv \frac{\max _{x} p(y \mid x)}{\max _{\{x, \nabla\}} p(y \mid x, \nabla)}=\frac{p\left(y \mid x_{0}\right)}{p\left(y \mid x_{a}, \nabla_{a}\right)}=\exp \left[-\frac{1}{2 \sigma^{2}}\left(r_{0}^{T} V^{-1} r_{0}-r_{a}^{T} V^{-1} r_{a}\right)\right],
$$

and the test statistic can be defined by

$$
\delta_{\mathrm{TS}} \equiv-2 \log _{e} \mathcal{L}\left(\mathrm{H}_{0}, \mathrm{H}_{a}\right)=\sigma^{-2}\left(r_{0}^{T} V^{-1} r_{0}-r_{a}^{T} V^{-1} r_{a}\right) \geq 0 .
$$


For some equivalent formulas for $\delta_{\mathrm{TS}}$, see [12, Sec 4.1]. It can be shown that (we illustrate this in Section 4, see (4.23))

$$
\begin{aligned}
\delta_{\mathrm{TS}} & \sim \chi^{2}(q, 0), \quad \text { under } \mathrm{H}_{0} ; \\
\delta_{\mathrm{TS}} & \sim \chi^{2}(q, \lambda), \quad \lambda=\sigma^{-4} \nabla^{T} C^{T} V^{-1} \operatorname{cov}\left\{r_{0}\right\} V^{-1} C \nabla, \quad \text { under } \mathrm{H}_{a},
\end{aligned}
$$

where $\operatorname{cov}\left\{r_{0}\right\}=\sigma^{2}\left[V-A\left(A^{T} V^{-1} A\right)^{-1} A^{T}\right]$. When $\delta_{\text {TS }}$ is larger than a given threshold, one decides to reject the null hypothesis $\mathrm{H}_{0}$ in favor of the alternative hypothesis $\mathrm{H}_{a}$. Otherwise the null hypothesis $\mathrm{H}_{0}$ will be accepted. The threshold is usually determined by the requirements of the specific application. For a discussion on this issue for general hypothesis testing, see for example [4, Sec 4.2.1] and [12, Sec 1.4].

3. Computational dangers. It is easy to assume that any reasonable method can be used to compute a simple result like $\delta_{\mathrm{TS}}$ in (2.8). However this is not true, and we give a small artificial example to illustrate some computational dangers. On the surface the following example looks fairly harmless, since the 2-norm condition numbers of $A$ and $V$ are about 4.44 and 33,000 respectively:

$$
\begin{gathered}
y=\left[\begin{array}{c}
5.48223618514353 \\
0.90878847962427 \\
25.94493985828999 \\
5.91432884267696
\end{array}\right], A=\left[\begin{array}{lll}
0.73591311187945 & 1.986990117078759 \\
0.01599725305719 & 1.85723508466859 \\
0.15632753551635 & 2.35754116764473 \\
0.65858764131884 & 0.21189908130823
\end{array}\right], C=\left[\begin{array}{l}
2.72281454895206 \\
1.87323437713309 \\
2.51387627488834 \\
0.87049065185808
\end{array}\right], \\
V=\left[\begin{array}{cccc}
9.140496886810 & -5.179920639550 & 22.018803142087 & -2.448166448348 \\
-5.179920639550 & 31.269615846900 & -38.726345506531 & 1.768700005165 \\
22.018803142087 & -38.726345506531 & 244.102164709880 & 43.463631186108 \\
-2.448166448348 & 1.768700005165 & 43.463631186108 & 15.497722556410
\end{array}\right], \quad \sigma^{2}=1 .
\end{gathered}
$$

This example was chosen to have $x_{0}=[1,2]^{T}$ and (see below) a large $\delta_{\mathrm{TS}}=\delta_{0}-\delta_{a}=$ $2-1=1$, which we would expect to be able to compute quite accurately. We carried out computations using Matlab 6 on an Apple Macintosh $450 \mathrm{MHz}$ PowerPC G4 running OSX 10.2.6, so the floating point relative precision was $\epsilon \approx 2.22 * 10^{-16}$. Now from (2.5) $x_{0}=\left(A^{T} V^{-1} A\right)^{-1} A^{T} V^{-1} y$, and $r_{0}=y-A x_{0}$, so

$$
\delta_{0} \equiv r_{0}^{T} V^{-1} r_{0}=y^{T} V^{-1} y-y^{T} V^{-1} A\left(A^{T} V^{-1} A\right)^{-1} A^{T} V^{-1} y .
$$

We computed this last expression using the Matlab code:

$$
\begin{aligned}
& \text { Vinv=inv(V); Atilde }=A^{\prime} * \text { Vinv; W=Atilde } * A \text {; } \\
& \text { delta_0=y'*(Vinv*y) }-y^{\prime} *(\text { Atilde' } *(\operatorname{inv}(\mathrm{W}) *(\text { Atilde } * y))) \text {; }
\end{aligned}
$$

and computed $\delta_{a} \equiv r_{a}^{T} V^{-1} r_{a}$ by replacing $A$ by $[A, C]$ in the above. From (2.8) we see $\delta_{\mathrm{TS}}=\delta_{0}-\delta_{a} \geq 0$. But our Matlab result was $\delta_{0}-\delta_{a}=-14.02906911842439$, an obviously nonsensical result. This was not caused by a fault in our programming, or in Matlab (our computations gave reasonable answers for all reasonable problems). It is a simple reminder that combining a sequence of seemingly reliable computations does not necessarily lead to an overall numerically stable computation.

The approach of computing $x_{0}$ above, then $r_{0}=y-A x_{0}$, then $\delta_{0} \equiv r_{0}^{T} V^{-1} r_{0}$, and similarly for $\delta_{a}$, gave $\delta_{0}-\delta_{a}=0.43885318681141$, which is meaningful, but still in error by more than $50 \%$. The numerically stable method we recommend in Section 4 gave $\delta_{\mathrm{TS}}=1.00000000078345$ and $x_{0}^{T}=$ [1.00000000000001 2.00000000000001]. Since a numerically stable computation gives the exact result to a nearby problem, see Section 5, we suspect that the above problem is reasonably well conditioned, and see that it is important to use a proven numerically stable algorithm for computing $\delta_{\mathrm{TS}}$. This is all the more important in many real time applications where we might not have the luxury of IEEE standard double precision floating point arithmetic.

The numerical instabilities of the above two "obvious" methods for $\delta_{\mathrm{TS}}$ were revealed here by $[A, C]$ having a 2 -norm condition number of about $1.6 * 10^{6}$. 
4. The algorithm. An obvious approach to solving problems GLLS $_{0}(2.5)$ and GLLS $_{a}(2.6)$ is to transform the problems to the corresponding ordinary LS problems. Suppose the symmetric positive definite $V$ has the factorization

$$
V=B B^{T},
$$

where $B \in \Re^{m \times m}$ is nonsingular. In some applications $B$ itself is given rather than $V$, then computing $V$ can result in loss of important information, and could cause the computed $V$ to be singular even when $B$ is nonsingular, see for example [1, p.163]. Even if $B$ is not given, we can compute the Cholesky factorization of $V$ to find $B$. Then by forming $B^{-1} y, B^{-1} A$ and $B^{-1} C,(2.5)$ and (2.6) can be transformed to

$$
\begin{array}{ll}
\operatorname{GLLS}_{0}: & \min \left(B^{-1} y-B^{-1} A x\right)^{T}\left(B^{-1} y-B^{-1} A x\right) ; \\
\operatorname{GLLS}_{a}: & \min \left(B^{-1} y-B^{-1} A x-B^{-1} C \nabla\right)^{T}\left(B^{-1} y-B^{-1} A x-B^{-1} C \nabla\right) .
\end{array}
$$

For these ordinary LS problems, the QR factorization method or the normal equation method can then be used to compute the optimal solutions and the test statistic $\delta_{\mathrm{TS}}$. This approach is still often used in some practical areas. But the solutions may unnecessarily lose accuracy when $B$ is ill-conditioned. Another problem is that for some applications the cofactor matrix $V$ may be singular, so that the GLLS formulations (2.5) and (2.6) (or (4.2) and (4.3)) and the test statistic $\delta_{\mathrm{TS}}$ in (2.8) are not defined. Fortunately we can use Paige's approach to solving GLLS problems (see [5]-[8]) to design a numerically stable method to compute the test statistic and the estimates of $x$ under $\mathrm{H}_{0}$ and under $\mathrm{H}_{a}$ as well. Our algorithm can handle a singular cofactor matrix $V$, but in order to expose the ideas quickly, we still assume that $V$ is nonsingular in this section and the next. We will show how to handle singular $V$ in Section 6 .

With (4.1), for the random noise vector $v$ in (2.1) and (2.2) we can write

$$
v \equiv B u, \quad u \sim \mathcal{N}\left(0, \sigma^{2} I\right) .
$$

Then the linear models (2.1) and (2.2) can be replaced by

$$
\begin{aligned}
& y=A x+B u, \quad u \sim \mathcal{N}\left(0, \sigma^{2} I\right), \quad \text { under } \mathrm{H}_{0} ; \\
& y=A x+C \nabla+B u, \quad u \sim \mathcal{N}\left(0, \sigma^{2} I\right), \quad \text { under } \mathrm{H}_{a} .
\end{aligned}
$$

Therefore problems GLLS in $_{0}$ (4.2) and $\mathrm{GLLS}_{a}$ in (4.3) can be reformulated as follows:

$$
\begin{array}{ll}
\operatorname{GLLS}_{0}: & \min _{u, x}\|u\|_{2}^{2} \text { subject to } y=A x+B u ; \\
\operatorname{GLLS}_{a}: & \min _{u, x, \nabla}\|u\|_{2}^{2} \text { subject to } y=A x+C \nabla+B u .
\end{array}
$$

Even if $B$ is not square or is singular, we can still find the optimal solutions for GLLS $\mathrm{S}_{0}$ and GLLS ${ }_{a}$ as long as the constraints in (4.7) and (4.8) are consistent (see Section 6).

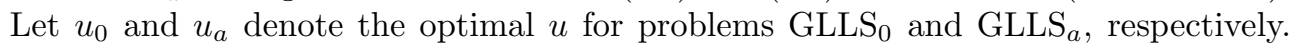
Then with the assumption that $B$ is nonsingular (see (2.7))

$$
u_{0}=B^{-1}\left(y-A x_{0}\right)=B^{-1} r_{0}, \quad u_{a}=B^{-1}\left(y-A x_{a}-C \nabla_{a}\right)=B^{-1} r_{a} .
$$

When $B=I, u_{0}$ and $u_{a}$ are just the ordinary LS residuals $r_{0}$ and $r_{a}$, respectively. So they can be called the generalized least squares (GLS) residuals. From (2.8), we have

$$
\delta_{\mathrm{TS}}=\sigma^{-2}\left(\left\|u_{0}\right\|_{2}^{2}-\left\|u_{a}\right\|_{2}^{2}\right) .
$$


Introducing $C \nabla$ decreases the square of the GLS residual norm from $\left\|u_{0}\right\|_{2}^{2}$ to $\left\|u_{a}\right\|_{2}^{2}$, so $\delta_{\mathrm{TS}}$ is a scaled measure of this decrease. Note that in the formulations (4.7), (4.8) and (4.10), no inverse of $B$ or $V$ appears. This is the key to the design of the numerically stable algorithm that we now develop.

First consider solving problem $\mathrm{GLLS}_{a}$ in (4.8). Let $[A, C]$ and $B$ have the following generalized QR (GQR) factorization (see [9])

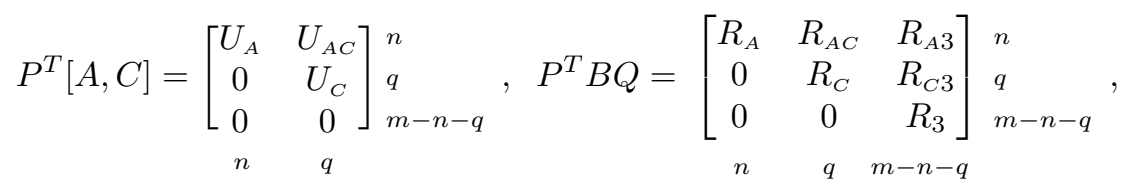

where

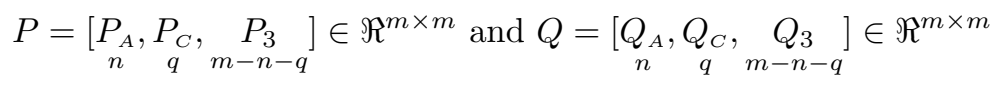

are orthogonal $\left(P^{-1}=P^{T}, Q^{-1}=Q^{T}\right)$, and $U_{A}, U_{C}, R_{A}, R_{C}$ and $R_{3}$ are nonsingular upper triangular. Multiplying the constraint equality in (4.8) by $P^{T}$ from the left and using $Q Q^{T}=I$, we have

$$
P^{T} y=P^{T} A x+P^{T} C \nabla+P^{T} B Q Q^{T} u \quad \text { under } \mathrm{H}_{a} .
$$

Then substituting (4.11) into the above equation and defining

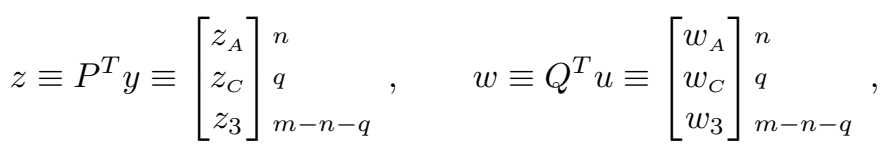

we can transform problem GLLS $_{a}$ in (4.8) to

$$
\begin{gathered}
\min \left(\left\|w_{A}\right\|_{2}^{2}+\left\|w_{C}\right\|_{2}^{2}+\left\|w_{3}\right\|_{2}^{2}\right) \text { subject to } \\
\left.\left[\begin{array}{c}
z_{A} \\
z_{C} \\
z_{3}
\end{array}\right]=\left[\begin{array}{c}
U_{A} \\
0 \\
0
\end{array}\right] x+\left[\begin{array}{c}
U_{A C} \\
U_{C} \\
0
\end{array}\right] \nabla+\left[\begin{array}{ccc}
R_{A} & R_{A C} & R_{A 3} \\
0 & R_{C} & R_{C 3} \\
0 & 0 & R_{3}
\end{array}\right]\left[\begin{array}{l}
w_{A} \\
w_{C} \\
w_{3}
\end{array}\right], \text { (i.e. under } \mathrm{H}_{a}\right) .
\end{gathered}
$$

Obviously the optimal solution $x_{a}, \nabla_{a}, w_{a} \equiv\left[\left(w_{A}^{a}\right)^{T},\left(w_{C}^{a}\right)^{T},\left(w_{3}^{a}\right)^{T}\right]^{T}$ satisfies

$$
w_{A}^{a}=0, \quad w_{C}^{a}=0, \quad\left[\begin{array}{ccc}
U_{A} & U_{A C} & R_{A 3} \\
0 & U_{C} & R_{C 3} \\
0 & 0 & R_{3}
\end{array}\right]\left[\begin{array}{c}
x_{a} \\
\nabla_{a} \\
w_{3}^{a}
\end{array}\right]=\left[\begin{array}{c}
z_{A} \\
z_{C} \\
z_{3}
\end{array}\right] \text {. }
$$

The upper triangular system in (4.15) can easily be solved by back substitution. From (4.14) and (4.15) we have $w_{3}^{a}=w_{3}$ under $\mathrm{H}_{a}$. Since $w=Q^{T} u \sim \mathcal{N}\left(0, \sigma^{2} I_{m}\right)$,

$$
w_{3}^{a} \sim \mathcal{N}\left(0, \sigma^{2} I_{m-n-q}\right), \quad \text { under } \mathrm{H}_{a}
$$

Thus $w_{3}^{a}$ is a vector of uncorrelated residuals with respect to the model (4.14), which can be regarded as the extension of the LUSH residuals (linear unbiased with scalar covariance matrix using Householder transformations) to a linear model with a general noise covariance matrix. For the LUSH residuals, see [10]. Thus the GLS residual $u_{a}$ (the optimal $u$ under $\mathrm{H}_{a}$ ) satisfies, see (4.13),

$$
u_{a}=Q w_{a}=Q_{3} w_{3}^{a}, \quad\left\|u_{a}\right\|_{2}^{2}=\left\|w_{a}\right\|_{2}^{2}=\left\|w_{A}^{a}\right\|_{2}^{2}+\left\|w_{C}^{a}\right\|_{2}^{2}+\left\|w_{3}^{a}\right\|_{2}^{2}=\left\|w_{3}^{a}\right\|_{2}^{2}
$$


Note that unlike the elements of $w_{3}^{a}$, the elements of $u_{a}$ are correlated, since from (4.16) and (4.17) $\operatorname{cov}\left\{u_{a}\right\}=\sigma^{2} Q_{3} Q_{3}^{T}$.

Now consider solving problem GLLS $_{0}$ in (4.7). Note that the difference between GLLS $_{0}$ and GLLS ${ }_{a}$ is that the constraint equality in GLLS ${ }_{0}$ does not have the $\nabla$ term. Thus from (4.14) it follows that GLLS $_{0}$ in (4.7) can be transformed to

$$
\begin{gathered}
\min \left(\left\|w_{A}\right\|_{2}^{2}+\left\|w_{C}\right\|_{2}^{2}+\left\|w_{3}\right\|_{2}^{2}\right), \\
\text { subject to }\left[\begin{array}{c}
z_{A} \\
z_{C} \\
z_{3}
\end{array}\right]=\left[\begin{array}{c}
U_{A} \\
0 \\
0
\end{array}\right] x+\left[\begin{array}{ccc}
R_{A} & R_{A C} & R_{A 3} \\
0 & R_{C} & R_{C 3} \\
0 & 0 & R_{3}
\end{array}\right]\left[\begin{array}{l}
w_{A} \\
w_{C} \\
w_{3}
\end{array}\right] .
\end{gathered}
$$

It follows that the optimal solution $x_{0}, w_{0} \equiv\left[\left(w_{A}^{0}\right)^{T},\left(w_{C}^{0}\right)^{T},\left(w_{3}^{0}\right)^{T}\right]^{T}$ satisfies

$$
w_{A}^{0}=0, \quad\left[\begin{array}{ccc}
U_{A} & R_{A C} & R_{A 3} \\
0 & R_{C} & R_{C 3} \\
0 & 0 & R_{3}
\end{array}\right]\left[\begin{array}{c}
x_{0} \\
w_{C}^{0} \\
w_{3}^{0}
\end{array}\right]=\left[\begin{array}{c}
z_{A} \\
z_{C} \\
z_{3}
\end{array}\right] .
$$

This upper triangular system can also be solved by back substitution. Note that the matrix coefficients and the right hand sides of both (4.15) and (4.19) were found from the one GQR in (4.11). Similar to $w_{3}^{a},\left[\begin{array}{l}w_{C}^{0} \\ w_{3}^{0}\end{array}\right]$ is a vector of uncorrelated residuals, since from (4.18), (4.19), (4.13) and (4.4)

$$
\left[\begin{array}{l}
w_{C}^{0} \\
w_{3}^{0}
\end{array}\right]=\left[\begin{array}{l}
w_{C} \\
w_{3}
\end{array}\right] \sim \mathcal{N}\left(0, \sigma^{2} I_{m-n}\right), \quad \text { under } \mathrm{H}_{0}
$$

Thus the GLS residual $u_{0}$ (the optimal $u$ under $\mathrm{H}_{0}$ ) satisfies

$$
u_{0}=Q w_{0}=Q_{C} w_{C}^{0}+Q_{3} w_{3}^{0}, \quad\left\|u_{0}\right\|_{2}^{2}=\left\|w_{0}\right\|_{2}^{2}=\left\|w_{C}^{0}\right\|_{2}^{2}+\left\|w_{3}^{0}\right\|_{2}^{2} .
$$

From (4.15) and (4.19) we observe that $w_{3}^{a}=w_{3}^{0}$. By using the equivalent formula of $\delta_{\mathrm{TS}}$ (see (4.10)), from (4.17) and (4.21) we obtain a result not involving differences:

$$
\delta_{\mathrm{TS}}=\sigma^{-2}\left(\left\|u_{0}\right\|_{2}^{2}-\left\|u_{a}\right\|_{2}^{2}\right)=\sigma^{-2}\left\|w_{C}^{0}\right\|_{2}^{2} .
$$

From (4.20) we see $w_{C}^{0} \sim \mathcal{N}\left(0, \sigma^{2} I_{q}\right)$ under $\mathrm{H}_{0}$, and we know $w_{C} \sim \mathcal{N}\left(0, \sigma^{2} I_{q}\right)$, see (4.13), (4.4). Combining (4.19) with (4.14) we have under $\mathrm{H}_{a}$,

$$
\left[\begin{array}{cc}
R_{C} & R_{C 3} \\
0 & R_{3}
\end{array}\right]\left[\begin{array}{l}
w_{C}^{0} \\
w_{3}^{0}
\end{array}\right]=\left[\begin{array}{c}
z_{C} \\
z_{3}
\end{array}\right]=\left[\begin{array}{c}
U_{C} \nabla \\
0
\end{array}\right]+\left[\begin{array}{cc}
R_{C} & R_{C 3} \\
0 & R_{3}
\end{array}\right]\left[\begin{array}{l}
w_{C} \\
w_{3}
\end{array}\right]
$$

so $w_{C}^{0}=R_{C}^{-1} U_{C} \nabla+w_{C} \sim \mathcal{N}\left(R_{C}^{-1} U_{C} \nabla, \sigma^{2} I_{q}\right)$ under $\mathrm{H}_{a}$. Therefore from (4.22) and the definition of the Chi-square distribution, we have

$$
\delta_{\mathrm{TS}} \sim \chi^{2}(q, 0) \quad \text { under } \mathrm{H}_{0} ; \quad \delta_{\mathrm{TS}} \sim \chi^{2}\left(q, \sigma^{-2}\left\|R_{C}^{-1} U_{C} \nabla\right\|_{2}^{2}\right) \quad \text { under } \mathrm{H}_{a} .
$$

It can be verified from (4.11) that the noncentrality parameter $\sigma^{-2}\left\|R_{C}^{-1} U_{C} \nabla\right\|_{2}^{2}$ is just the $\lambda$ given in (2.9).

The above derivation leads to the following algorithm to compute the test statistic, the BLUE $x_{0}$ under $\mathrm{H}_{0}$ and the BLUE $x_{a}$ under $\mathrm{H}_{a}$. 


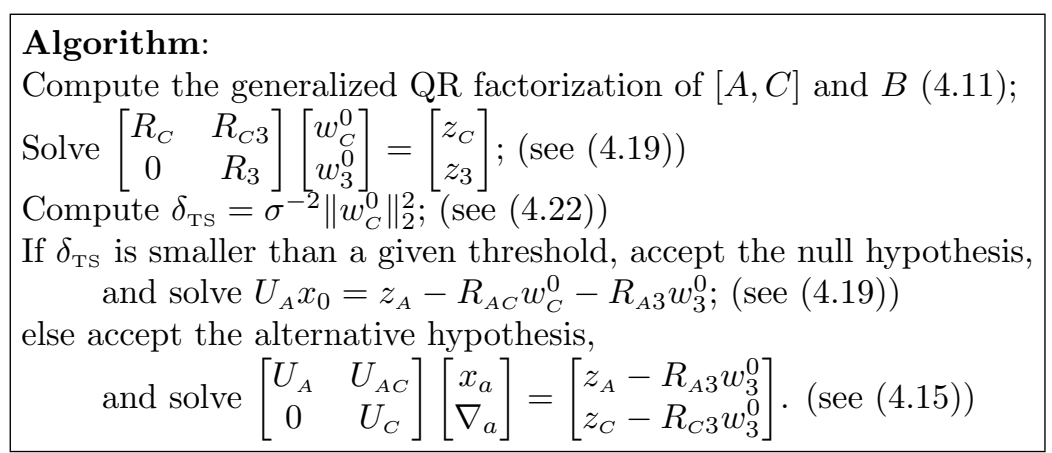

In the following we give a few comments about the above algorithm.

The GQR factorization (4.11) can be computed using Householder transformations. $P$ and $Q$ are not needed to be formed in the computation. Note that $R_{A}$ is not involved in the computation. Thus it is not required to be upper triangular, or even square $\left(R_{A}\right.$ may not be square when $B$ is not square, see Section 6$)$. After we compute the QR factorization of $A$, we apply $P^{T}$ to $B$ and obtain

$$
P^{T} B=\left[\begin{array}{ll}
P_{A}^{T} & B \\
P_{C}^{T} & B \\
P_{3}^{T} & B
\end{array}\right]
$$

Then we determine $Q$ by the RQ factorization:

$$
\left[\begin{array}{l}
P_{C}^{T} B \\
P_{3}^{T} B
\end{array}\right] Q=\left[\begin{array}{ccc}
0 & R_{C} & R_{C 3} \\
0 & 0 & R_{3}
\end{array}\right]
$$

Although both $R_{A C}$ and $R_{A 3}$ are involved in computing $x_{0}$, and $R_{A 3}$ is involved in computing $x_{a}$, we do not need to explicitly compute them. In fact what we need to compute are $R_{A C} w_{C}^{0}+R_{A 3} w_{3}^{0}$ and $R_{A 3} w_{3}^{0}$. Note that by (4.11)

$$
R_{A C} w_{C}^{0}+R_{A 3} w_{3}^{0}=P_{A}^{T} B Q\left[\begin{array}{c}
0 \\
w_{C}^{0} \\
w_{3}^{0}
\end{array}\right], \quad R_{A 3} w_{3}^{0}=P_{A}^{T} B Q\left[\begin{array}{c}
0 \\
0 \\
w_{3}^{0}
\end{array}\right],
$$

where $P_{A}^{T} B$ has been computed in (4.24). The above computation can be performed by using only matrix-vector multiplications.

Sometimes one is interested in the covariance matrices $\operatorname{cov}\left\{x_{0}\right\}$ under $\mathrm{H}_{0}$ and $\operatorname{cov}\left\{x_{a}\right\}$ under $\mathrm{H}_{a}$. First we consider computing $\operatorname{cov}\left\{x_{0}\right\}$ under $\mathrm{H}_{0}$. From (4.14) without the $\nabla$ term on the right hand side, and (4.19), we obtain

$$
\left[\begin{array}{ccc}
U_{A} & R_{A C} & R_{A 3} \\
0 & R_{C} & R_{C 3} \\
0 & 0 & R_{3}
\end{array}\right]\left[\begin{array}{c}
x_{0}-x \\
w_{C}^{0}-w_{C} \\
w_{3}^{0}-w_{3}
\end{array}\right]=\left[\begin{array}{c}
R_{A} w_{A} \\
0 \\
0
\end{array}\right]
$$

This shows that $w_{3}^{0}-w_{3}=0$ and $w_{C}^{0}-w_{C}=0$, so

$$
U_{A}\left(x_{0}-x\right)=R_{A} w_{A} .
$$

Since $w=Q^{T} u \sim \mathcal{N}\left(0, \sigma^{2} I\right)$, see (4.13) and (4.4), we have

$$
U_{A} \cdot \operatorname{cov}\left\{x_{0}\right\} \cdot U_{A}^{T}=\sigma^{2} R_{A} R_{A}^{T} .
$$


We see that here $R_{A}$ is needed, although it is not needed in computing the test statistic and the estimates $x_{0}$ and $x_{a}$. If $\operatorname{cov}\left\{x_{0}\right\}$ will be involved in other computations, it might be useful to have an upper triangular $R_{A}$. Notice if $R_{A}$ is upper triangular, $\sigma U_{A}^{-1} R_{A}$ is the Cholesky factor of $\operatorname{cov}\left\{x_{0}\right\}$. Computing this, however, could lead to loss of accuracy if $U_{A}$ is ill-conditioned. In many applications we may not need to compute $\operatorname{cov}\left\{x_{0}\right\}$ explicitly. In general (4.25) gives the most reliable representation of $\operatorname{cov}\left\{x_{0}\right\}$.

Now we consider computing $\operatorname{cov}\left\{x_{a}\right\}$ under $\mathrm{H}_{a}$. From (4.14) and (4.15) we obtain

$$
\left[\begin{array}{ccc}
U_{A} & U_{A C} & R_{A 3} \\
0 & U_{C} & R_{C 3} \\
0 & 0 & R_{3}
\end{array}\right]\left[\begin{array}{c}
x_{a}-x \\
\nabla_{a}-\nabla \\
w_{3}^{a}-w_{3}
\end{array}\right]=\left[\begin{array}{cc}
R_{A} & R_{A C} \\
0 & R_{C} \\
0 & 0
\end{array}\right]\left[\begin{array}{l}
w_{A} \\
w_{C}
\end{array}\right]
$$

This shows that $w_{3}^{a}-w_{3}=0$, so

$$
\left[\begin{array}{cc}
U_{A} & U_{A C} \\
0 & U_{C}
\end{array}\right]\left[\begin{array}{c}
x_{a}-x \\
\nabla_{a}-\nabla
\end{array}\right]=\left[\begin{array}{cc}
R_{A} & R_{A C} \\
0 & R_{C}
\end{array}\right]\left[\begin{array}{l}
w_{A} \\
w_{C}
\end{array}\right]
$$

Again since $w \sim \mathcal{N}\left(0, \sigma^{2} I\right)$, we have the representation of $\operatorname{cov}\left\{\left[\begin{array}{c}x_{a} \\ \nabla_{a}\end{array}\right]\right\}$ :

$$
\left[\begin{array}{cc}
U_{A} & U_{A C} \\
0 & U_{C}
\end{array}\right] \cdot \operatorname{cov}\left\{\left[\begin{array}{c}
x_{a} \\
\nabla_{a}
\end{array}\right]\right\} \cdot\left[\begin{array}{cc}
U_{A} & U_{A C} \\
0 & U_{C}
\end{array}\right]^{T}=\sigma^{2}\left[\begin{array}{cc}
R_{A} & R_{A C} \\
0 & R_{C}
\end{array}\right]\left[\begin{array}{cc}
R_{A} & R_{A C} \\
0 & R_{C}
\end{array}\right]^{T}
$$

and $\operatorname{cov}\left\{x_{a}\right\}$ can be found by solving this. If we want to get the reliable representation of $\operatorname{cov}\left\{x_{a}\right\}$ similar to (4.25), we find two orthogonal matrices $G_{1}$ and $G_{2}$ such that

$$
G_{1}^{T}\left[\begin{array}{cc}
U_{A} & U_{A C} \\
0 & U_{C}
\end{array}\right]=\left[\begin{array}{cc}
\tilde{U}_{A} & 0 \\
\tilde{U}_{C A} & \tilde{U}_{C}
\end{array}\right], \quad G_{1}^{T}\left[\begin{array}{cc}
R_{A} & R_{A C} \\
0 & R_{C}
\end{array}\right] G_{2}=\left[\begin{array}{cc}
\tilde{R}_{A} & 0 \\
\tilde{R}_{C A} & \tilde{R}_{C}
\end{array}\right],
$$

where $G_{1}$ is a product of Givens rotations, $G_{2}$ is a product of Householder transformations, and $\tilde{U}_{A}$ and $\tilde{R}_{A}$ are still upper triangular. This computation can be implemented to make full use of the structures of the relevant matrices for efficiency. Combining (4.26) and (4.27) shows

$$
\left[\begin{array}{cc}
\tilde{U}_{A} & 0 \\
\tilde{U}_{C A} & \tilde{U}_{C}
\end{array}\right] \cdot \operatorname{cov}\left\{\left[\begin{array}{c}
x_{a} \\
\nabla_{a}
\end{array}\right]\right\} \cdot\left[\begin{array}{cc}
\tilde{U}_{A} & 0 \\
\tilde{U}_{C A} & \tilde{U}_{C}
\end{array}\right]^{T}=\sigma^{2}\left[\begin{array}{cc}
\tilde{R}_{A} & 0 \\
\tilde{R}_{C A} & \tilde{R}_{C}
\end{array}\right]\left[\begin{array}{cc}
\tilde{R}_{A} & 0 \\
\tilde{R}_{C A} & \tilde{R}_{C}
\end{array}\right]^{T}
$$

The $(1,1)$ block of the two sides of this equality gives the representation of $\operatorname{cov}\left\{x_{a}\right\}$ :

$$
\tilde{U}_{A} \cdot \operatorname{cov}\left\{x_{a}\right\} \cdot \tilde{U}_{A}^{T}=\sigma^{2} \tilde{R}_{A} \tilde{R}_{A}^{T} .
$$

5. Numerical stability of the algorithm. In this section we will prove the numerical stability of the computation of the test statistic $\delta_{\mathrm{TS}}$, the BLUE $x_{0}$ of $x$ under $\mathrm{H}_{0}$ and the BLUE $x_{a}$ under $\mathrm{H}_{a}$. The proof extends the approach in [7].

Let the computed triangular matrices in the GQR factorization (4.11) be denoted by $\hat{U}$ and $\hat{R}$. We can show (see for example [2, Lem. 18.3, Thm. 18.4]) that there exist orthogonal $\tilde{P} \in \Re^{m \times m}$ and $\tilde{Q} \in \Re^{m \times m}$ such that

$$
\begin{aligned}
& \tilde{P}^{T}\left[A+\Delta A_{1}, C+\Delta C_{1}\right]=\left[\begin{array}{cc}
\hat{U}_{A} & \hat{U}_{A C} \\
0 & \hat{U}_{C} \\
0 & 0
\end{array}\right], \\
& \left\|\Delta A_{1}\right\|_{F}=O(\epsilon)\|A\|_{F}, \quad\left\|\Delta C_{1}\right\|_{F}=O(\epsilon)\|A\|_{F},
\end{aligned}
$$


10 XIAO-WEN CHANG, CHRISTOPHER C. PAIGE, AND CHRISTIAN C.J.M. TIBERIUS

$$
\tilde{P}^{T}\left(B+\Delta B_{1}\right) \tilde{Q}=\left[\begin{array}{ccc}
\hat{R}_{A} & \hat{R}_{A C} & \hat{R}_{A 3} \\
0 & \hat{R}_{C} & \hat{R}_{C 3} \\
0 & 0 & \hat{R}_{3}
\end{array}\right], \quad\left\|\Delta B_{1}\right\|_{F}=O(\epsilon)\|A\|_{F},
$$

where $\epsilon$ is the unit roundoff, and for simplicity we use $O(\epsilon)$ instead of the product of $\epsilon$ and a constant dependent only on the dimensions of the problem, and here and later we ignore any $O\left(\epsilon^{2}\right)$ terms.

Note that $z=P^{T} y$ (see $\left.(4.13)\right)$. We can show that the computed $z$ satisfies (see for example [2, Lem. 18.3])

$$
\hat{z}=\tilde{P}^{T}(y+\Delta y), \quad\|\Delta y\|_{2}=O(\epsilon)\|y\|_{2},
$$

and the computed solution $\hat{x}_{0}, \hat{w}_{C}^{0}, \hat{w}_{3}^{0}$ of (4.19) satisfies (see [2, Lem. 8.5])

$$
\begin{gathered}
{\left[\begin{array}{ccc}
\hat{U}_{A}+\Delta U_{A} & \hat{R}_{A C}+\Delta R_{A C} & \hat{R}_{A 3}+\Delta R_{A 3} \\
0 & \hat{R}_{C}+\Delta R_{C} & \hat{R}_{C 3}+\Delta R_{C 3} \\
0 & 0 & \hat{R}_{3}+\Delta R_{3}
\end{array}\right]\left[\begin{array}{c}
\hat{x}_{0} \\
\hat{w}_{C}^{0} \\
\hat{w}_{3}^{0}
\end{array}\right]=\left[\begin{array}{c}
\hat{z}_{A} \\
\hat{z}_{C} \\
\hat{z}_{3}
\end{array}\right],} \\
\left\|\Delta U_{A}\right\|_{F}=O(\epsilon)\left\|\hat{U}_{A}\right\|_{F}, \quad\left\|\left[\begin{array}{cc}
\Delta R_{A C} & \Delta R_{A 3} \\
\Delta R_{C} & \Delta R_{C 3} \\
0 & \Delta R_{3}
\end{array}\right]\right\|_{F}=O(\epsilon)\left\|\left[\begin{array}{cc}
\hat{R}_{A C} & \hat{R}_{A 3} \\
\hat{R}_{C} & \hat{R}_{C 3} \\
0 & \hat{R}_{3}
\end{array}\right]\right\|_{F} .
\end{gathered}
$$

For the computation of $\delta_{\mathrm{TS}}=\sigma^{-2}\left\|w_{C}^{0}\right\|_{2}^{2}$, it is easy to show that the computed $\delta_{\mathrm{TS}}$ satisfies (see [2, p. 69])

$$
\hat{\delta}_{\mathrm{TS}}=(\sigma+\Delta \sigma)^{-2}\left\|\hat{w}_{C}^{0}\right\|_{2}^{2}, \quad \Delta \sigma=O(\epsilon) \sigma .
$$

Combining (5.1), (5.2), (5.3) and (5.4), we can easily verify that $\hat{\delta}_{\mathrm{TS}}$ and $\hat{x}_{0}$ are the exact test statistic and the BLUE of $x$ under $\mathrm{H}_{0}$, respectively, for the initial data

$$
\begin{aligned}
& \tilde{y} \equiv y+\Delta y, \quad\|\tilde{y}-y\|_{2}=O(\epsilon)\|y\|_{2}, \\
& \tilde{A} \equiv A+\Delta A_{1}+\tilde{P}\left[\begin{array}{c}
\Delta U_{A} \\
0 \\
0
\end{array}\right], \quad\|\tilde{A}-A\|_{F}=O(\epsilon)\|A\|_{F}, \\
& \tilde{B} \equiv B+\Delta B_{1}+\tilde{P}\left[\begin{array}{cc}
\Delta R_{A C} & \Delta R_{A 3} \\
\Delta R_{C} & \Delta R_{C 3} \\
0 & \Delta R_{3}
\end{array}\right] \tilde{Q}^{T}, \quad\|\tilde{B}-B\|_{F}=O(\epsilon)\|B\|_{F}, \\
& \tilde{\sigma} \equiv \sigma+\Delta \sigma, \quad|\tilde{\sigma}-\sigma|=O(\epsilon)|\sigma| .
\end{aligned}
$$

Thus the computations of $\delta_{\mathrm{TS}}$ and $x_{0}$ are numerically stable. It is pleasing that $\hat{\delta}_{\mathrm{TS}}$ and $\hat{x}_{0}$ are exact for initial data with the same small perturbations.

Similarly we can easily show the computation of the BLUE $x_{a}$ of $x$ under $\mathrm{H}_{a}$ is numerically stable.

6. Extension to the singular case. In this section we assume that the rank of the cofactor matrix $V$ is $k \leq m$. Suppose $V$ still has the factorization (4.1), where $B \in \Re^{m \times k}$ has full column rank. If $B$ is not given, we can use diagonal pivoting to compute the Cholesky factorization of $V$ (see for example $[1, \S 2.2 .2$.$] ) to give (4.1),$ where the strictly upper triangular part of $B$ is zero, i.e., $b_{i j}=0$ for $i<j$, or the 'reverse' Cholesky factorization of $V$ to give (4.1), where $b_{i j}=0$ for $i>m-k+j$. So we still have models (4.5) and (4.6). 
If $y \notin \mathcal{R}([A, B])$ then $y$ could not have come from the model (2.1), and this has to be checked. For simplicity we assume that the two models are consistent, so that unique optimal solutions $\left\{x_{0}, u_{0}\right\}$ and $\left\{x_{a}, \nabla_{a}, u_{a}\right\}$ exist for problems GLLS $_{0}$ in (4.7) and $\mathrm{GLLS}_{a}$ in (4.8), respectively. Now (see [5, §3]), the solution of GLLS $\mathrm{S}_{0}$ results in the BLUE of any estimable function of $x$ under $\mathrm{H}_{0}$. Also since $B$ has full column rank we have $B^{\dagger} B=I$, and writing $v=B u$ in (2.1) we see that in (4.5) and (4.6)

$$
u=B^{\dagger} v \sim \mathcal{N}\left(0, \sigma^{2} I_{k}\right) .
$$

From this we will show that we can still use the formula for the test statistic $\delta_{\mathrm{TS}}$ in (4.10). Unlike $u_{0}$ and $u_{a}$ in (4.9), $y=A x_{0}+B u_{0}$ etc. lead to

$$
u_{0}=B^{\dagger}\left(y-A x_{0}\right), \quad u_{a}=B^{\dagger}\left(y-A x_{a}-C \nabla_{a}\right) .
$$

In the following we will make a sequence of orthogonal transformations to compute GQR factorizations involving $A, C$ and $B$. Based on these, we then discuss the computation of $\delta_{\mathrm{TS}}, x_{0}$ and $x_{a}$.

Just as in (4.11), we find the QR factorization of $[A, C]$ :

$$
\bar{P}^{T}[A, C]=\frac{\left[\begin{array}{cc}
U_{A} & U_{A C} \\
0 & U_{C} \\
0 & 0
\end{array}\right]_{m-n-q}^{n} .}{n}
$$

Then apply $\bar{P}^{T}$ to $B$ to get

$$
\bar{P}^{T} B=\left[\begin{array}{l}
B_{A} \\
B_{C} \\
B_{3}
\end{array}\right] \begin{aligned}
& n \\
& m-n-q
\end{aligned}
$$

$k$

We would like to transform $B_{3}$ to a nonsingular upper triangular matrix. First find the RQ factorization of $B_{3}$ with row pivoting:

$$
\Pi_{3}^{T} B_{3} Q=\underset{k-l}{\left[\begin{array}{cc}
0 & \bar{R}_{3}
\end{array}\right] m-n-q}
$$

where $\Pi_{3}$ is a permutation matrix, $Q$ is orthogonal, and $\bar{R}_{3}$ is a full-column-rank trapezoidal matrix with zeros in its strictly lower left triangle. Then find the QR factorizations of $\bar{R}_{3}$ :

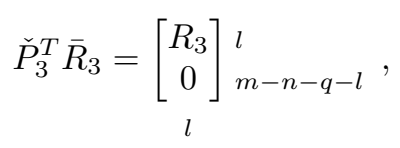

where $\check{P}_{3}$ is orthogonal and $R_{3}$ is nonsingular upper triangular. Combining (6.4) and (6.5) leads to

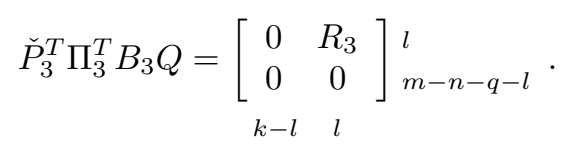


Defining

$$
P \equiv \bar{P}\left[\begin{array}{ccc}
I_{n} & 0 & 0 \\
0 & I_{q} & 0 \\
0 & 0 & \Pi_{3} \check{P}_{3}
\end{array}\right],
$$

we then have from $(6.2),(6.3)$ and (6.6) that

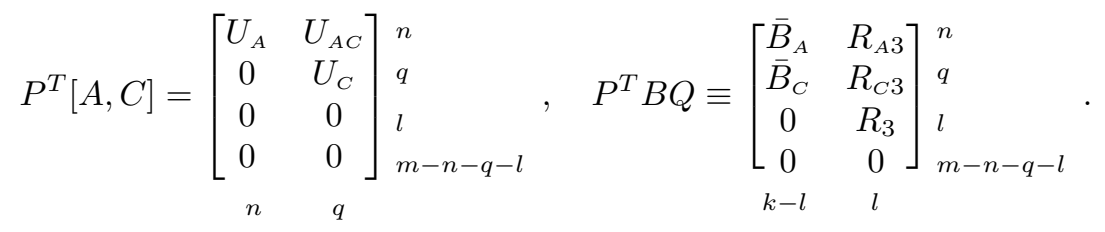

Here $\bar{B}_{C}$ does not have any special structure.

As in Section 4, defining

$$
z \equiv P^{T} y \equiv\left[\begin{array}{c}
z_{A} \\
z_{C} \\
z_{3} \\
z_{4}
\end{array}\right]_{m-n-q-l}^{n}, \quad w \equiv Q^{T} u \equiv\left[\begin{array}{c}
w_{A C} \\
w_{3}
\end{array}\right]_{l}^{k-l},
$$

we transform problem GLLS in $_{a}(4.8)$ to $(c f .(4.14))$

$$
\begin{gathered}
\min \left(\left\|w_{A C}\right\|_{2}^{2}+\left\|w_{3}\right\|_{2}^{2}\right) \\
\text { subject to }\left[\begin{array}{c}
z_{A} \\
z_{C} \\
z_{3} \\
z_{4}
\end{array}\right]=\left[\begin{array}{c}
U_{A} \\
0 \\
0 \\
0
\end{array}\right] x+\left[\begin{array}{c}
U_{A C} \\
U_{C} \\
0 \\
0
\end{array}\right] \nabla+\left[\begin{array}{cc}
\bar{B}_{A} & R_{A 3} \\
\bar{B}_{C} & R_{C 3} \\
0 & R_{3} \\
0 & 0
\end{array}\right]\left[\begin{array}{c}
w_{A C} \\
w_{3}
\end{array}\right] .
\end{gathered}
$$

Here the $\nabla$ term will disappear when we transform problem GLLS $_{0}$ in (4.7) by the same transformations. Note that $z_{4}$ in (6.9) must be a zero vector, since the original model (4.6) is consistent. Obviously the optimal solution $x_{a}, \nabla_{a}, w_{a} \equiv$ $\left[\left(w_{A C}^{a}\right)^{T},\left(w_{3}^{a}\right)^{T}\right]^{T}$ satisfies $(c f .(4.15))$

$$
w_{A C}^{a}=0, \quad\left[\begin{array}{ccc}
U_{A} & U_{A C} & R_{A 3} \\
0 & U_{C} & R_{C 3} \\
0 & 0 & R_{3}
\end{array}\right]\left[\begin{array}{c}
x_{a} \\
\nabla_{a} \\
w_{3}^{a}
\end{array}\right]=\left[\begin{array}{c}
z_{A} \\
z_{C} \\
z_{3}
\end{array}\right] .
$$

Then it follows that the GLS residual $u_{a}$ (the optimal $u$ under $\mathrm{H}_{a}$ ) satisfies

$$
u_{a}=Q w_{a}, \quad\left\|u_{a}\right\|_{2}^{2}=\left\|w_{a}\right\|_{2}^{2}=\left\|w_{3}^{a}\right\|_{2}^{2} .
$$

Now we consider solving problem GLLS $_{0}$ in (4.7). From (6.9) we observe that GLLS $_{0}$ can be transformed to

$$
\begin{gathered}
\min \left(\left\|w_{A C}\right\|_{2}^{2}+\left\|w_{3}\right\|_{2}^{2}\right) \\
\text { subject to }\left[\begin{array}{c}
z_{A} \\
z_{C} \\
z_{3}
\end{array}\right]=\left[\begin{array}{c}
U_{A} \\
0 \\
0
\end{array}\right] x+\left[\begin{array}{cc}
\bar{B}_{A} & R_{A 3} \\
\bar{B}_{C} & R_{C 3} \\
0 & R_{3}
\end{array}\right]\left[\begin{array}{c}
w_{A C} \\
w_{3}
\end{array}\right] .
\end{gathered}
$$


Note that (6.12) is just (6.9) except that the $\nabla$ term and $z_{4}$ rows have been removed. In order to estimate $x$, we have to transform $\bar{B}_{C}$ to a nonsingular upper triangular matrix. As we did to $B_{3}$ (see (6.6)), we can find orthogonal $\check{P}_{C} \in \Re^{q \times q}$ and $\check{Q}_{C} \in$ $\Re^{(k-l) \times(k-l)}$, and a permutation $\Pi_{C} \in \Re^{q \times q}$ such that

$$
\begin{array}{rl}
\check{P}_{C}^{T} \Pi_{C}^{T} \bar{B}_{C} \check{Q}_{C}= & {\left[\begin{array}{cc}
0 & R_{C} \\
0 & 0
\end{array}\right]_{q-p}^{p},} \\
k-l-p & p
\end{array}
$$

where $R_{C}$ is nonsingular upper triangular. Let

$$
\begin{aligned}
& \check{P}_{C}^{T} \Pi_{C}^{T} z_{C} \equiv\left[\begin{array}{l}
\check{z}_{C} \\
\tilde{z}_{C}
\end{array}\right]_{q-p}^{p}, \quad \check{P}_{C}^{T} \Pi_{C}^{T} R_{C 3} \equiv\left[\begin{array}{l}
\check{R}_{C 3} \\
\tilde{R}_{C 3}
\end{array}\right]{ }_{q-p}^{p}, \\
& \left.\bar{B}_{A} \check{Q}_{C} \equiv \underset{k-l-p}{\underset{R_{A}}{R_{A}}, R_{A C}}\right] n, \quad \check{Q}_{C}^{T} w_{A C} \equiv\left[\begin{array}{l}
w_{A} \\
w_{C}
\end{array}\right] \begin{array}{l}
k-l-p \\
p
\end{array} .
\end{aligned}
$$

Therefore from (6.12) with (6.13)-(6.15) we observe that problem GLLS $_{0}$ can be further transformed to

$$
\begin{gathered}
\min \left(\left\|w_{A}\right\|_{2}^{2}+\left\|w_{C}\right\|_{2}^{2}+\left\|w_{3}\right\|_{2}^{2}\right) \\
\text { subject to }\left[\begin{array}{c}
z_{A} \\
\check{z}_{C} \\
\tilde{z}_{C} \\
z_{3}
\end{array}\right]=\left[\begin{array}{c}
U_{A} \\
0 \\
0 \\
0
\end{array}\right] x+\left[\begin{array}{ccc}
R_{A} & R_{A C} & R_{A 3} \\
0 & R_{C} & \check{R}_{C 3} \\
0 & 0 & \tilde{R}_{C 3} \\
0 & 0 & R_{3}
\end{array}\right]\left[\begin{array}{l}
w_{A} \\
w_{C} \\
w_{3}
\end{array}\right] .
\end{gathered}
$$

Notice that the bottom block equation in (6.16) can uniquely determine the estimate of $w_{3}$, since $R_{3}$ is nonsingular. Since the equations in (6.16) are consistent, the third block equation can be removed. Then obviously the optimal solution $x_{0}, w_{0} \equiv$ $\left[\left(w_{A}^{0}\right)^{T},\left(w_{C}^{0}\right)^{T},\left(w_{3}^{0}\right)^{T}\right]^{T}$ satisfies

$$
w_{A}^{0}=0, \quad\left[\begin{array}{ccc}
U_{A} & R_{A C} & R_{A 3} \\
0 & R_{C} & \check{R}_{C 3} \\
0 & 0 & R_{3}
\end{array}\right]\left[\begin{array}{c}
x_{0} \\
w_{C}^{0} \\
w_{3}^{0}
\end{array}\right]=\left[\begin{array}{c}
z_{A} \\
z_{C} \\
z_{3}
\end{array}\right] .
$$

Then from the second equality in (6.8) and the second equality in (6.15), we observe that the GLS residual $u_{0}$ (the optimal $u$ under $\mathrm{H}_{0}$ ) satisfies

$$
u_{0} \equiv Q\left[\begin{array}{c|c}
\check{Q}_{C} & 0 \\
\hline 0 & I
\end{array}\right]\left[\begin{array}{c}
w_{A}^{0} \\
w_{C}^{0} \\
\hline w_{3}^{0}
\end{array}\right], \quad\left\|u_{0}\right\|_{2}^{2}=\left\|w_{C}^{0}\right\|_{2}^{2}+\left\|w_{3}^{0}\right\|_{2}^{2} .
$$

As in Section $4, w_{3}^{a}=w_{3}^{0}$ (see (6.10) and (6.17)), so using the formula of the test statistic in (4.10) we have from (6.11) and (6.18) that

$$
\delta_{\mathrm{TS}}=\sigma^{-2}\left(\left\|u_{0}\right\|_{2}^{2}-\left\|u_{a}\right\|_{2}^{2}\right)=\sigma^{-2}\left\|w_{C}^{0}\right\|_{2}^{2},
$$

so that once again introducing $C \nabla$ decreases the square of the GLS residual from $\left\|u_{0}\right\|_{2}^{2}$ to $\left\|u_{a}\right\|_{2}^{2}$, and $\delta_{\mathrm{TS}}$ is a scaled measure of this decrease.

Now we look at the distribution of $\delta_{\mathrm{TS}}$. Using the fact that orthogonal transformations of a unit covariance random vector do not change its covariance matrix, we see from (6.16) and (6.17) that $w_{C}^{0}=w_{C}$, and from (6.15), (6.8) and (6.1) that

$$
w_{C}^{0} \sim \mathcal{N}\left(0, \sigma^{2} I_{p}\right), \quad \text { under } \mathrm{H}_{0} .
$$


Under $\mathrm{H}_{a}$ the transformed model has the form (6.9). Let (cf. (6.14))

$$
\check{P}_{C}^{T} \Pi_{C}^{T} U_{C} \equiv\left[\begin{array}{c}
\check{U}_{C} \\
\tilde{U}_{C}
\end{array}\right] \begin{aligned}
& p \\
& q-p
\end{aligned},
$$

then (6.9) with the $z_{4}$ rows removed can be transformed to (cf. (6.16))

$$
\left[\begin{array}{c}
z_{A} \\
\tilde{z}_{C} \\
\tilde{z}_{C} \\
z_{3}
\end{array}\right]=\left[\begin{array}{c}
U_{A} \\
0 \\
0 \\
0
\end{array}\right] x+\left[\begin{array}{c}
U_{A C} \\
\tilde{U}_{C} \\
\tilde{U}_{C} \\
0
\end{array}\right] \nabla+\left[\begin{array}{ccc}
R_{A} & R_{A C} & R_{A 3} \\
0 & R_{C} & \check{R}_{C 3} \\
0 & 0 & \tilde{R}_{C 3} \\
0 & 0 & R_{3}
\end{array}\right]\left[\begin{array}{c}
w_{A} \\
w_{C} \\
w_{3}
\end{array}\right] .
$$

Then from (6.17) and (6.20) we see $R_{C} w_{C}^{0}=\check{z}_{C}=\check{U}_{C} \nabla+R_{C} w_{C}$, so

$$
w_{C}^{0} \sim \mathcal{N}\left(R_{C}^{-1} \check{U}_{C} \nabla, \sigma^{2} I_{p}\right), \quad \text { under } \mathrm{H}_{a} .
$$

Thus $\delta_{\text {TS }}$ in (6.19) is the desired test statistic, since (cf. (4.23))

$$
\delta_{\mathrm{TS}} \sim \chi^{2}(p, 0) \quad \text { under } \mathrm{H}_{0} ; \quad \delta_{\mathrm{TS}} \sim \chi^{2}\left(p, \sigma^{-2}\left\|R_{C}^{-1} \check{U}_{C} \nabla\right\|_{2}^{2}\right) \quad \text { under } \mathrm{H}_{a} .
$$

From (6.13) we see that $p$ is the rank of $\bar{B}_{C}$, and this can be determined in computing (6.13). Here we give a theoretical formula for it. Suppose for $P$ in (6.7)

$$
P \equiv\left[P_{1}, P_{2}, P_{3}\right] \text { such that }\left[\frac{P_{2}^{T}}{P_{3}^{T}}\right] B Q=\left[\begin{array}{cc}
\bar{B}_{C} & R_{C 3} \\
0 & R_{3} \\
0 & 0
\end{array}\right]_{m-n-q-l}^{q} l_{l}^{q} .
$$

Thus it follows that

$$
\operatorname{rank}\left(\left[P_{2}, P_{3}\right]^{T} B Q\right)=\operatorname{rank}\left(\bar{B}_{C}\right)+\operatorname{rank}\left(R_{3}\right), \quad \operatorname{rank}\left(P_{3}^{T} B Q\right)=\operatorname{rank}\left(R_{3}\right)=l .
$$

But

$$
\operatorname{rank}\left(\left[P_{2}, P_{3}\right]^{T} B Q\right)=\operatorname{rank}\left(\left(P_{2} P_{2}^{T}+P_{3} P_{3}^{T}\right) B\right), \quad \operatorname{rank}\left(P_{3}^{T} B Q\right)=\operatorname{rank}\left(P_{3} P_{3}^{T} B\right),
$$

and from the QR factorization of $[A, C]$ in (6.7)

$$
P_{2} P_{2}^{T}+P_{3} P_{3}^{T}=I-A A^{\dagger}, \quad P_{3} P_{3}^{T}=I-[A, C][A, C]^{\dagger} .
$$

Substituting (6.22) and (6.23) into (6.21), we obtain

$$
p=\operatorname{rank}\left(\bar{B}_{C}\right)=\operatorname{rank}\left(\left(I-A A^{\dagger}\right) B\right)-\operatorname{rank}\left(\left(I-[A, C][A, C]^{\dagger}\right) B\right) .
$$

Notice that when $B$ is nonsingular,

$$
p=\operatorname{rank}\left(I-A A^{\dagger}\right)-\operatorname{rank}\left(I-[A, C][A, C]^{\dagger}\right)=(m-n)-(m-n-q)=q .
$$

This is the case we discussed in Section 4.

We could discuss the computation of the covariance matrices for the estimates in the same way as we did in Section 4. But for brevity we omit this.

Since the theory and algorithm here can handle a singular noise covariance matrix, we can now easily handle linear equality constraints without needing to develop a new theory or algorithm. Suppose the two hypotheses are

$$
\begin{array}{ll}
\mathrm{H}_{0}: & y=A x+v, \quad v \sim \mathcal{N}\left(0, \sigma^{2} V\right), \quad \text { subject to } \quad E x=d ; \\
\mathrm{H}_{a}: & y=A x+C \nabla+v, \quad v \sim \mathcal{N}\left(0, \sigma^{2} V\right), \quad \text { subject to } \quad E x=d,
\end{array}
$$


where $[A, C]$ has full column rank and the symmetric nonnegative definitive $V$ has the factorization (4.1) with $B$ being of full column rank. Then we can apply our algorithm directly to the following GLLS problems to find the test statistic and the estimates as well:

$$
\begin{array}{llll}
\operatorname{GLLS}_{0}: & \min \|u\|_{2}^{2} & \text { subject to } & {\left[\begin{array}{l}
y \\
d
\end{array}\right]=\left[\begin{array}{l}
A \\
E
\end{array}\right] x+\left[\begin{array}{c}
B \\
0
\end{array}\right] u} \\
\operatorname{GLLS}_{a}: & \min \|u\|_{2}^{2} & \text { subject to } & {\left[\begin{array}{l}
y \\
d
\end{array}\right]=\left[\begin{array}{l}
A \\
E
\end{array}\right] x+\left[\begin{array}{c}
C \\
0
\end{array}\right] \nabla+\left[\begin{array}{c}
B \\
0
\end{array}\right] u .}
\end{array}
$$

Note that $\left[\begin{array}{l}A \\ E\end{array}\right],\left[\begin{array}{cc}A & C \\ E & 0\end{array}\right]$ and $\left[\begin{array}{l}B \\ 0\end{array}\right]$ have full column rank.

7. Summary. The standard formula for the generalized likelihood ratio test statistic is not suitable for numerical computation when any of $A,[A, C]$, or the noise covariance matrix $\sigma^{2} V$ in (2.1) and (2.2) is ill-conditioned. Also the formula is not defined when the noise covariance matrix is singular. To overcome these problems, we gave a new formula for the test statistic after reformulating the two GLLS problems for estimating the parameter vectors. The new formula is mathematically equivalent to the standard one when the noise covariance matrix is nonsingular, and is well defined when the noise covariance matrix is singular. With this formula, a numerically stable algorithm based on Paige's GLLS method was proposed to compute it. The computations of the test statistic and the BLUEs of the parameter vectors are closely connected - they are obtained from the solutions of two upper triangular linear equations, (4.15) and (4.19). We also showed how to compute reliable representations of the error covariance matrices for the BLUEs, see (4.25) and (4.26) et seq.. In our algorithm, we did not assume that $B$ has any special structure. If $B$ comes from the Cholesky factorization of the noise covariance matrix, then we can use the structure of $B$ to design a faster algorithm by following the approach in [8].

\section{REFERENCES}

[1] A, BлÖвск, Numerical Methods for Least Squares Problems, SIAM Publication, Philadelphia, PA, 1996.

[2] N. J. Higham, Accuracy and Stability of Numerical Algorithms, Society for Industrial and Applied Mathematics, Philadelphia, PA, 1996.

[3] B. Hofmann-Wellenhof, H. Lichtenegger, and J. Collins, GPS Theory and Practice, 4th ed., Springer, New York, 1997.

[4] K. R. Koch, Parameter Estimation and Hypothesis Testing in Linear Models, 2nd ed., Springer, Berlin, 1999.

[5] S. Kourouklis and C. C. Paige, A constrained least squares approach to the general GaussMarkov linear model, J. Amer. Statist. Assoc., 76 (1981), pp. 620-625.

[6] C. C. PAIge, Numerical stable computations for general univariate linear models, Comm. Statist., B7 (1978), pp. 437-453.

[7] C. C. PAige, Computer Solution and Perturbation Analysis of Generalized Linear Least Squares Problems, Math. Comput., 33 (1979), pp. 171-183.

[8] C. C. PAige, Fast Numerically Stable Computations for Generalized Linear Least Squares Problems, SIAM J. Num. Anal., 16 (1979), pp. 165-171.

[9] C. C. Paige, Some Aspects of Generalized QR Factorizations, in Reliable Numerical Computation, Cox M.G., Hammarling S. (eds), Oxford University Press, New York, pp. 73-91, 1990.

[10] G. P. H. StYAn, LUSH and other uncorrelated residuals from linear models, Canadian Journal of Statistics, 1 (1973), pp. 131-132.

[11] P. J. Teunissen, Quality Control and GPS, in GPS for Geodesy, Teunissen P.J., Kleusberg A. (eds), 2nd edn. Springer, Berlin, pp. 271-318, 1998.

[12] P. J. Teunissen, Testing Theory, An Introduction, Delft University Press, Delft, 2000. 